\section{Is Cheater/Cooperator Detection an In-Group Phenomenon? Some Preliminary Findings}

\author{
Jan Verplaetse ${ }^{1, *}$, Sven Vanneste ${ }^{2}$ \\ 1. Department of Jurisprudence and Legal History, The Moral \\ Brain, Ghent University, 9000 Ghent, Belgium. \\ 2. Brai²n, TRI \& Department of Neurosurgery, University Hospital \\ Antwerp, 3000 Antwerp, Belgium. \\ *Author for correspondence (ian.verplaetse@ugent.be
}

Whereas predictive detection of (non-)cooperative intentions among humans is well-documented, virtually nothing is known about the cross-cultural extent of this possibly evolved social intuition. In this study we asked Caucasian participants to judge Japanese subjects who played a trust game in which they either fairly divided the money (sharer) or kept the entire sum (non-sharer). After watching 5-seconds videotapes taken around decision Caucasian subjects were able to discriminate non-sharing and sharing Japanese targets slightly above chance level (51.71\%). The non-sharers accuracy rate was $52.32 \%$ and the sharers accuracy rate was $51.10 \%$, but significant higher than would be expected from randomly guessing alone. This preliminary finding suggests that successful cheater/cooperator detection is not limited to own-culture targets and questions the in-group nature of this social intuition.

\section{Keywords}

cheater/cooperator detection, trust game, crosscultural

\section{Introduction}

Psychological research has convincingly shown that humans are able to detect non-cooperative or cooperative intentions from thin slices of facial information (Brown, Palameta, \& Moore, 2003; Frank, Gilovich, \& Regan, 1993; Oda, Naganawa, Yamauchi, Yamagata, \& Matsumoto-Oda, 2009; Pradel, Euler, \& Fetchenhauer, 2009; Verplaetse, Vanneste, \& Braeckman, 2007). For instance, Verplaetse et al. (2007) found that $66 \%$ of the noncooperative players in a one-shot prisoner's dilemma game could be correctly identified if raters were confronted with a photograph taken at the very moment of their decision. Other research teams obtained similar results. A Japanese research group (Yamagishi, Shinada, Kiyonari, \& Schug, 2009) asked students to watch videotaped decisions of tar- gets playing a trust game during which they could either allocate money equally or keep the entire sum for themselves. Again, raters were able to judge the strangers' cooperativeness higher than chance (58\%), although this time fair allocators were more accurately identified. This culture-independent finding suggests that humans are equipped with intuitive skills to avoid cheaters and/or select cooperative partners in prospect of risky cooperation. Since cheater/cooperator detection skills are essential to the mechanism of reciprocal altruism, these findings lend support to the evolutionary hypothesis that reciprocal altruism was of crucial importance for making trustworthiness decisions in ancestral human communities (Hirschleifer, 1987; Trivers, 1971).

However, very little is known about the crosscultural extent of cheater/cooperator detection. To start investigating this issue, we simply asked Caucasian people to judge the cooperativeness of Japanese targets. Initially, we expected a significant decline in detection accuracy (Mesquita \& Frijda, 1992). Prior cross-cultural research demonstrated a cultural effect in the recognition and expression of emotions. For instance, people seem better at recognizing own-culture emotional expressions relative to other-culture emotional expressions (Elfenbein \& Ambady, 2002; Marsh, Elfenbein, \& Ambady, 2003). Additionally, culture shapes how and when emotions are expressed. Cultural-specific rules govern when it is appropriate to express particular emotions, explaining why emotional control might be the norm in one culture (such as in Japan), while overt expression of emotions might be the standard in the other (such as in Europe) (Masuda et al., 2008; Matsumoto et al., 1998; Yuki, Maddux, \& Masuda, 2007). Since cheater/cooperator detection dominantly relies on the expression and recognition of subtle emotional cues, one might expect that the detection skills of Caucasion subjects do not work well in response to Japanese targets. Moreover, anthropologists argued that social interaction among humans in the distant past was limited to individuals with whom we have the most contact, so that strangers would likely not have been trusted in any case (Schmidt \& Cohn, 2001). If our ability to detect defective intentions is the outcome of an evolutionary process dating back to our ancestral environment, successful identification of cheaters/cooperators is restricted to people from our own culture.

Nevertheless, other research puts this in-group bias into perspective. Without ignoring cultural influences, basic emotions theory emphasizes that there is an underlying innate mechanism that allows universal recognition and expression to occur initially. Emotions are equivalent in their physical 
Table 1. One-Sample t-test for the Accuracy Rate

\begin{tabular}{|c|c|c|}
\hline Target 5-seconds movies & Accuracy rate \% (SD) & p-value \\
\hline Overall & (7.12) & $<10$ \\
\hline $\begin{array}{l}\text { Sharers } \\
\text { Non-sharers }\end{array}$ & $(8.12)$ & $<.01$ \\
\hline Male targets & & \\
\hline Sharers & $(9.08)$ & $n s$. \\
\hline Non-sharers & $(10.26)$ & $<.001$ \\
\hline $\begin{array}{l}\text { Female targets } \\
\text { Sharers } \\
\text { Non-Sharers }\end{array}$ & $\begin{array}{l}(9.83) \\
(9.59)\end{array}$ & $\begin{array}{l}<.05 \\
n s .\end{array}$ \\
\hline
\end{tabular}

signalling properties and produce high, well-abovechance agreements in recognition levels across different cultures (Ekman \& Friesen, 1971; Ekman, Sorenson, \& Friesen, 1969). According to this line of research, diminished levels in cross-cultural emotion recognition are either superficial or attributable to factors irrelevant to the recognition process itself (Biehl et al., 1997; Matsumoto, 1992, 2007; Matsumoto et al., 2002). Even if emotional recognition of own-culture targets reaches optimal levels, the overall finding that we accurately identify emotions from other-culture targets must prevail. Consequently, one might reason that cheater/cooperator detection relies upon more universal expressions and gestures, which are untouched by culture-depending rules. Furthermore, one may doubt whether cheater/cooperator detection is limited to targets whom we frequently met. Predictive cheater detection is most useful in situations where we encounter potential partners for the first time and when no reputation information is available. If the reputation of the partner is unknown because he or she belongs to another group or culture, we must screen his or her social intentions from expressive facial cues in advance (Verplaetse, 2008). With respect to these considerations, membership of a different culture is just another kind of unfamiliarity, inherent to all exchange opportunities with unknown partners. Accordingly, our ability to discriminate cheaters from cooperators will work equally well in response to targets from another culture.

Taking both lines of reasoning into account, we expected that Caucasian subjects discern Japanese non-cooperators from cooperators above chance level, but possibly less successful with regard to the original, mono-cultural studies. We hypothesize that cultural specificity weakens the subjects' performance in terms of accuracy without entirely wiping out the basic social intuition to detect cheaters/cooperators.

\section{Method}

We asked 128 students (85 females; age $18.5 \pm 1.79$ ) during a class at Ghent University (Belgium) to watch 5 -seconds movies delivered by our Japanese colleagues from Hokkaido University (Sapporo, Japan). The Hokkaido research group (Yamagishi et al., 2009) previously videotaped the decisions of undergraduates at Hokkaido University who were invited to play a one-shot trust game with an anonymous partner. The targets could either divide the amount of money in a fair manner (300 yen to both parties) or behave selfish and keep the entire sum of money (600 yen for target). All trials were videotaped using a visible web camera in order to make 5-seconds movies that captured the targets' expression and gestures during the actual decision. Of course, the actual decision itself was hidden from view. The Hokkaido researchers randomly selected the 5 -seconds movies of 54 male targets and 48 female targets. Half of the female targets and half of the male targets shared the money with the unknown partner. This way, sharers and nonshares were equally balanced. Finally, although audio was turned off during recordings, an offscreen bell sound indicated the moment of decision. So, observers had an exact idea when the target actually decided during the 5 -seconds fragments.

Before showing the Japanese targets we explained the trust game to our mainly Belgian and Caucasian subjects. Using a paper-and-pencil test they were asked to judge whether the target was either a sharer or not and how sure they were about there particular judgment (5-point Likert scale; 1 $=$ totally not sure and $5=$ totally sure). Additional items gauged their overall performance estimation (5-point Likert scale; $1=$ very easy and $5=$ very difficult). In advance we also communicated that the proportion of sharers/non-sharers was equal and that a bell sound indicated the targets' decision moment. To encourage participation and attention a reward was promised and given to the best performer (50 euros). We presented the male targets first and without pauses between the target movies. Subsequently, we showed the female targets in an equal manner.

Statistical analyses were performed using SPSS software package. One-sample t-tests were conducted to determine whether the various identification rates exceeded chance level (50\%). Paired t-tests were preformed to verify whether there were differences in accuracy rates between sharers and nonsharers.

\section{Results}

The responses of 5 participants could not be used due to omissions. Also, we omitted the responses of 4 non-Caucasian students from further analysis. The overall accuracy rate was $51.71 \%(\mathrm{SD}=4.72)$ and significantly exceeds chance level, t(118) = $3.96, \mathrm{p}<.001$. The non-sharers accuracy rate was $52.32 \%(\mathrm{SD}=8.12)$ and the sharers accuracy rate was $51.10 \%(\mathrm{SD}=7.12)$, both are significant higher than would be expected from randomly guessing 


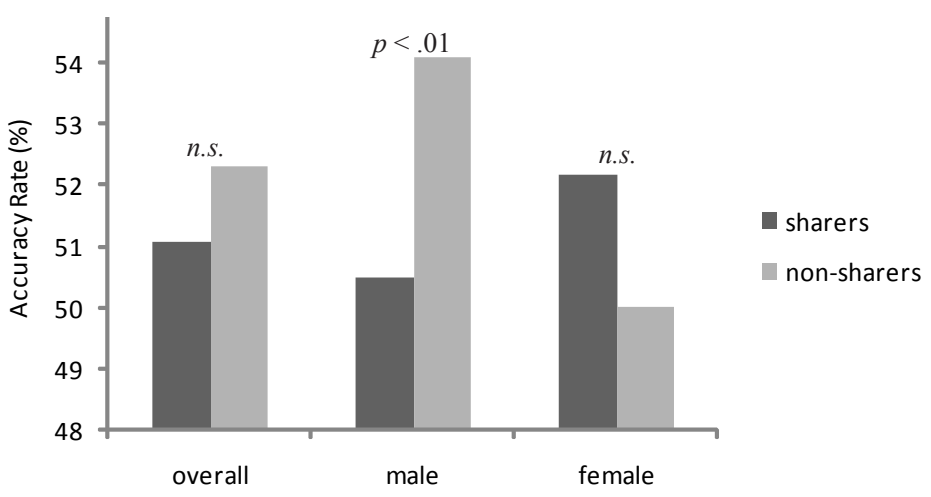

Figure 1. Accuracy rate for respectively overall (male + female), male and female targets

alone, respectively $\mathrm{t}(118)=3.12, \mathrm{p}<.01$ and $\mathrm{t}(118)=$ $1.68, \mathrm{p}<.10$. However, the difference between both rates was not significant, $\mathrm{t}(118)=1.13, \mathrm{p}=.27$, so we are not allowed to attribute a general cheater or cooperator bias to our subjects. See Table 1 for an overview.

If we focus on the targets' gender, we noticed a higher accuracy rate for male non-sharers $(\mathrm{M}=$ $54.12 \%$; $\mathrm{SD}=10.26$ ) and a higher accuracy rate for female sharers $(52.19 \%$; SD $=9.83)$. Both accuracy percentages significantly exceeded chance level, respectively $\mathrm{t}(118)=4.38, \mathrm{p}<.001$ and $\mathrm{t}(118)=2.43$, $\mathrm{p}<.05$. The accuracy rate for male sharers $(50.00 \%$; $\mathrm{SD}=9.08)$ and female non-sharers $(50.52 \%$; $\mathrm{SD}=$ 9.59) did not exceed chance level, respectively t(118) $=.01, \mathrm{p}=.99$ and $\mathrm{t}(118)=.60, \mathrm{p}=.55$. Analyses yielded a significant difference between sharers and non-sharers for male targets, $\mathrm{t}(118)=3.08, \mathrm{p}<.01$, but not for female targets, $\mathrm{t}(118)=1.34, \mathrm{p}=.18$ ( $\mathrm{see}$ figure 1). These results suggest a cheater bias towards male targets among Caucasian subjects.

However, prudence is required. Despite our communication that sharers and non-sharers were equally balanced, there was a slight tendency among our participants to overestimate the male targets as non-sharers $(52.06 \% ; \mathrm{SD}=7.28)$ and the female targets $(50.83 \%$; $\mathrm{SD}=6.81)$ as sharers. $\mathrm{Nev}^{-}$ ertheless, if we compare the accuracy rate and frequency rate of male non-sharers, $t(118)=3.52, p<$ .011 and the accuracy rate and frequency rate of $\mathrm{fe}^{-}$ male sharers $\mathrm{t}(118)=2.14, \mathrm{p}<.05$, we still hold significant effects. This implies that the above-chance detection of male non-sharers and female sharers cannot be explained by a certain bias or a general stereotype that males are mostly non-sharers and females mostly sharers.

In general, participants were not that confident about their decision $(M=2.44$; $S D=1.52)$. No category yielded significant differences. Finally, participants found the detection task rather difficult $(\mathrm{M}=$ $4.02 ; \mathrm{SD}=.86$ ), although they understood the task very well.

\section{Discussion}

This study suggests that Caucasian subjects might be able to judge the social intentions of Japanese individuals above chance level. Watching Japanese undergraduates who were videotaped during a 5 seconds movie while playing a trust game, gave our subjects some clue whether a player shared the money with his partner or not to a certain extent. This result argues against the idea that cheater/ cooperator detection must be considered an in-group phenomenon, as cross-cultural studies in emotion expression and recognition and anthropological theory on deception put forward (Schmidt \& Cohn, 2001). However, compared with the results obtained in the original, mono-cultural studies the accuracy rates are far from impressive. While in the original studies $66 \%$ of cheaters (Verplaetse et al., 2007) and $58 \%$ of the cooperators (Yamagishi et al., 2009) were on average correctly classified, the accuracy rate in this cross-cultural study dropped to $52.32 \%$ (non-sharers) and 51.10\% (sharers). Although these mean percentages exceeded chance level, an ingroup-advantage seemed present as well. Detection rates presumably increase when subjects are confronted with own-culture faces relative to otherculture faces and vice versa.

We like to emphasize the preliminary nature of these findings. First, our conclusions would be more convincing if Japanese subjects judged our Caucasian pictures set as well. Since this work remains to be done, our claim concerning a cross ${ }^{-}$ cultural intuition to detect defectors/cooperators is partial at best. Moreover, caution is required to claim an in-group advantage since we never asked our subjects to judge videotaped Caucasian subjects when they make their decision in a trust game. Our comparison ignores possibly detection differences between photographed PDG players and videotaped trust game players. Second, further investigation without prior announcement about equal composition of targets is most wanted. Besides more realistic, such a design will yield information about the levels of trust or distrust towards out-group members. These levels might affect the accuracy rates. If Caucasian subjects for instance estimate more non-cooperative behaviour among Japanese players, then the quantity of correctly identified cheaters will increase as well. Yet, a higher 
detection rate does not automatically prove a cross ${ }^{-}$ cultural intuition to identify cheaters. They might just be more distrustful in response to foreigners. Third, our Caucasian raters were not punished for making a wrong decision, what runs against the logic of cheater/cooperator detection. More complex designs should be introduced to probe the influence of punishment and reward on the subjects' detection skills (for instance, see Oda et al., 2009). Finally, given that our findings are preliminary it would be rash to interpret and theorise about the cheater bias towards male targets. Anyhow, it is interesting to notice that we could not replicate the cooperators bias found in the original Japanese study (Yamagishi et al., 2009). In response to male targets we actually found a cheater bias.

To our knowledge this is the first study in cheater detection using a cross-cultural design. We found that Caucasians subject are able to discern Japanese sharers from non-sharers whom they never met before slightly above chance level. However, in stead of considering this finding a final conclusion that settles the issue, we see this research as an initial impetus for more crosscultural research on cheater/cooperator detection.

\section{Acknowledgement}

We thank Toshio Yamagishi and Mizuho Shinada for sharing the stimulus material with us.

\section{References}

Biehl, M., Matsumoto, D., Ekman, P., Hearn, V., Heider, K., Kudoch, T., \& Ton, V. (1997). Matsumoto and Ekman's Japanese and Caucasian Facial Expressions of Emotions (JACFEE): Reliability data and cross-national differences. Journal of Nonverbal Behavior, 21, 3-21.

Brown, W. M., Palameta, B., \& Moore, C. (2003). Are there nonverbal cues to commitment? An exploratory study using the zero-acquaintance video presentation paradigm. Evolutionary Psychology, 1, 42-69.

Ekman, P., \& Friesen, W. V. (1971). Constants across cultures in the face and emotion. Journal of Personality and Social Psychology, 17, 124129.(doi:10.1037/h0030377)

Ekman, P., Sorenson, E. R., \& Friesen, W. V. (1969). Pan-cultural elements in facial displays of emotion. Science, 164, 86-88. (doi: 10.1126/science.164.3875.86)

Elfenbein, H. A., \& Ambady, N. (2002). On the universality and cultural specificity of emotion recognition: A meta-analysis. Psychological Bulletin, 128, 203-235. (doi: 10.1037//0033. 2909.128.2.203

Frank, R. H., Gilovich, T., \& Regan, D. T. (1993). The evolution of one-shot cooperation: An experiment. Ethology and Sociobiology, 14, 247256. (doi 10.1016/0162-3095(93)90020-I)

Hirshleifer, J. (1987). On the emotions as guarantors of threats and promises. In J. Dupré (Ed.), The Latest on the Best: Essays on evolution and optimality (pp. 307-326). Cambridge: MIT.
Marsh, A. A., Elfenbein, H. A., \& Ambady, N. (2003). Nonverbal "accents": Cultural differences in facial expression of emotion. Psychological Science, $14,373-376$.

Masuda, T., Mesquita, B., Tanida, S., Ellsworth, P. C., Leu, J., \& Van de Veerdonk, E. (2008). Placing the face in context: Cultural differences in the perception of facial emotions. Journal of Personality and Social Psychology, 94, 365-381. (doi: 10.1037/0022-3514.94.3.365)

Matsumoto,D. (1992). American-Japanese cultural differences in the recognition of universal facial expressions. Journal of Cross-Cultural Psychology, 23, 72-84. (doi:10.1177/0022022192231005)

Matsumoto, D. (2007). Emotion judgments do not differ as a function of perceived nationality. International Journal of Psychology, 42, 207214.(doi 10.1080/00207590601050926)

Matsumoto, D., Consolacion, T., Yamada, H., Suzuki, R., Franklin, B., Paul, S., ... Uchida, H. (2002). American-Japanese cultural differences in judgments of emotional expressions of different intensities. Cognition \& Emotion, 16, 721747. (doi $10.1080 / 02699930143000608)$

Matsumoto, D., Takeuchi, S., Andayani, S., Kouznetsova, N., \& Krupp, D. (1998) The contribution of individualism vs. collectivism to cross-national differences in display rules. Asian Journal of Social Psychology, 1, 147-165.

Mesquita, B., \& Frijda, N. H. (1992). Cultural variation in emotion: A review. Psychological Bulletin, 112, 179-204.

Oda, R., Naganawa, T., Yamauchi, S., Yamagata, N., \& Matsumoto-Oda, A. (2009). Altruists are trusted based on non-verbal cues. Biology Letters, 5, 752-754. (doi: 10.1098/rsbl.2009.0332)

Pradel, J., Euler, H. A., \& Fetchenhauer, D. (2009). Spotting altruistic dictator game players and mingling with them: the elective assortation of classmates. Evolution and Human Behavior, 30, 103-113. (doi:10.1016 i.evolhumbehav.2008.09.003)

Schmidt, K. L., \& Cohn, J. F. (2001). Human facial expressions as adaptations: Evolutionary questions in facial expression research. Yearbook of Physical Anthropology, 44, 3-24. (doi: 10.1002 ajpa.2001)

Trivers, R. L. (1971) The evolution of reciprocal altruism. Quarterly Review of Biology, 46, 35-55. (doi: 10.1086/406755)

Verplaetse, J. (2008). Het morele instinct. Over de biologische oorsprong van onze moraal [The moral instinct. On the biological origin of our moral sense]. Amsterdam: Nieuwezijds.

Verplaetse, J., Vanneste, S., \& Braeckman, J. (2007). You can judge a book by its cover: The sequel. A kernel of evolutionary truth in predictive cheating detection. Evolution and Human Behavior, 28, 260-271. (doi:10.1016) i.evolhumbehav.2007.04.006)

Yamagishi, T., Shinada, M., Kiyonari, T., \& Schug, J. (2009, March). Can people detect a stranger's trustworthiness in the zero-acquaintance situation. Paper presented at the Workshop on Cheater/Cooperator Detection, the $17^{\text {th }}$ International Workshop at Hokkaido University, 
Sapporo, Japan.

Yuki, M., Maddux, W. W., \& Masuda, T. (2007).

Are the windows to the soul the same in the East and West? Cultural differences in using eyes and mouth as cues to recognize emotions in Japan and the United States. Journal of Experimental Social Psychology, 43, 303-311. (doi: 10.1016/i.jesp.2006.02.004 